\title{
3D MODELING BY CONSOLIDATION OF INDEPENDENT GEOMETRIES EXTRACTED FROM POINT CLOUDS. THE CASE OF THE MODELING OF THE TURCKHEIM'S CHAPEL (ALSACE, FRANCE).
}

\author{
M. Koehl ${ }^{a,}{ }^{*}-$ Ph. Fabre ${ }^{a}-$ B. Schlussel ${ }^{b}$ \\ ${ }^{\text {a }}$ Laboratoire ICube - UMR 7357, INSA de Strasbourg, F-67084 Strasbourg, France \\ (mathieu.koehl, philippe.fabre @ insa-strasbourg.fr) \\ ${ }^{\mathrm{b}}$ Société d'Histoire de la ville de Turckheim (F-68320) \\ (schlussel @ turckheim.fr)
}

\section{Commission V}

KEY WORDS: 3D Modeling, 3D CAD, geometric consolidation, cultural heritage

\section{ABSTRACT :}

Turckheim is a small town located in Alsace, north-east of France.

In the heart of the Alsatian vineyard, this city has many historical monuments including its old church. To understand the effectiveness of the project described in this paper, it is important to have a look at the history of this church. Indeed there are many historical events that explain its renovation and even its partial reconstruction.

The first mention of a christian sanctuary in Turckheim dates back to 898. It will be replaced in the 12th century by a roman church (chapel), which subsists today as the bell tower. Touched by a lightning in 1661, the tower then was enhanced. In 1736, it was repaired following damage sustained in a tornado. In 1791, the town installs an organ to the church. Last milestone, the church is destroyed by fire in 1978. The organ, like the heart of the church will then have to be again restored (1983) with a simplified architecture.

From this heavy and rich past, it unfortunately and as it is often the case, remains only very few documents and information available apart from facts stated in some sporadic writings. And with regard to the geometry, the positioning, the physical characteristics of the initial building, there are very little indication.

Some assumptions of positions and right-of-way were well issued by different historians or archaeologists. The acquisition and 3D modeling project must therefore provide the current state of the edifice to serve as the basis of new investigations and for the generation of new hypotheses on the locations and historical shapes of this church and its original chapel (Fig.1)

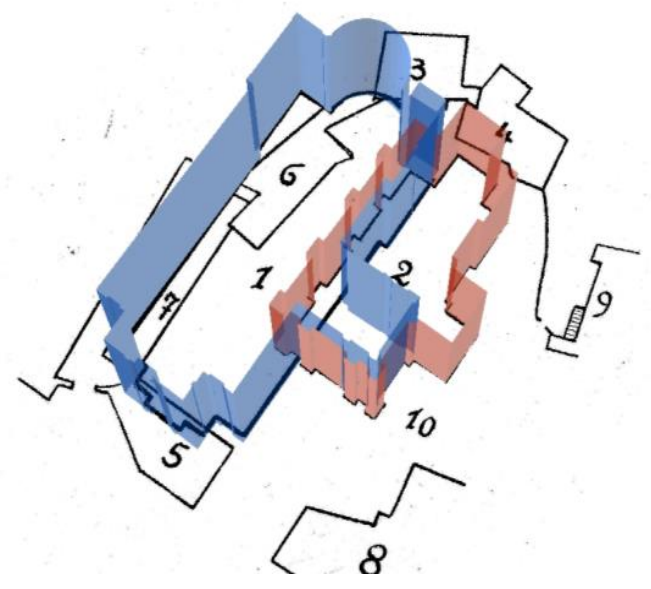

Fig.1: Hypothetic localization of chapel (2) vs actual church (1)

To enable the modeling, topography and TLS were used for inner and outer survey.

The modeling phase has allowed to test a new modeling process. Instead start by consolidating the point clouds in a single one, then extract segmentations for fine modeling by parts, we proceed to light modeling from each point cloud regarded initially as an independent one. Working in a highly sophisticated 3D CAD environment (Bentley Microstation) allows one hand to integrate every point clouds (in the pointools .pod format) and, from there, to model the different parts in independent geometries, either by digitizing construction and adjustment of primitives to point cloud. 
As different point clouds overlap, the modeled parts will also have parts in recovery. The final model can be obtained by consolidation, not of point clouds, but by adjustment of geometric elements between them (using the recovery parts). Each part is so referenced in the general system.

Basic architecture of the recently reconstructed part is thus easily transposable in a 3D model.

The oldest part, and in particular residues of the chapel can be modeled more finely, or even as detailed meshed parts derived from point clouds in the special case of the remains of the chapel.

A structuring in levels and an association to a database could also accompany this process of modeling to use it later in an information system and particular to distinguish the different constituent elements.

The integration of the final model in the complete cloud allows showing differences between the resulting model and the consolidated point cloud and validates the modeling approach. In particular the aspects of speed execution within a powerful CAD environment and its availability of a lot of primitives and construction methods as well as the geometric precision of the final method will be discussed.

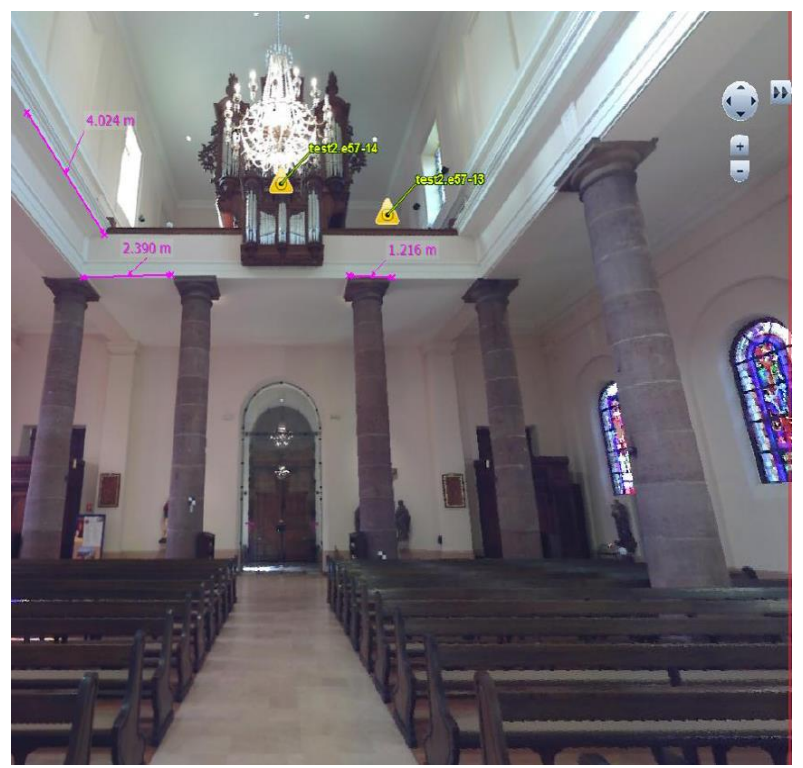

Fig. 2: Church interior as point cloud with measurement functions

In parallel and for better readability and sharing of knowledge on the building, we also realized a virtual tour through panoramic images (Fig. 2) as well as from the consolidated point clouds (Fig. 3 and Fig. 4).

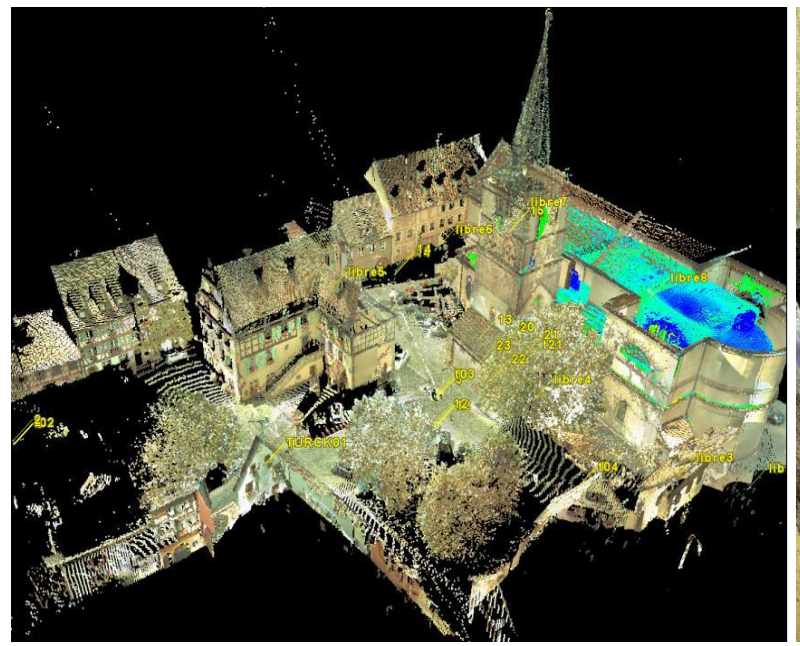

Fig. 3: Global consolidated point clouds

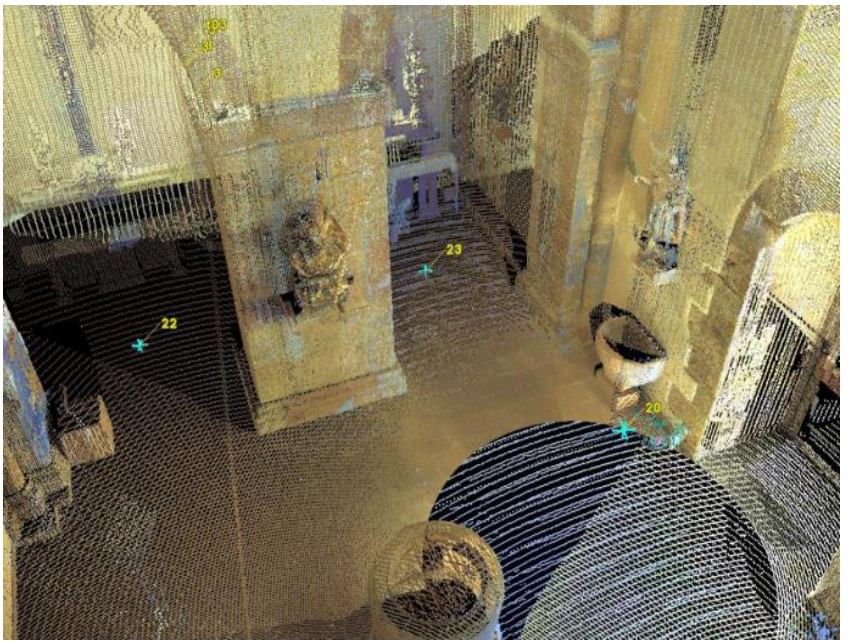

Fig. 4: Extract of chapel point cloud with details 


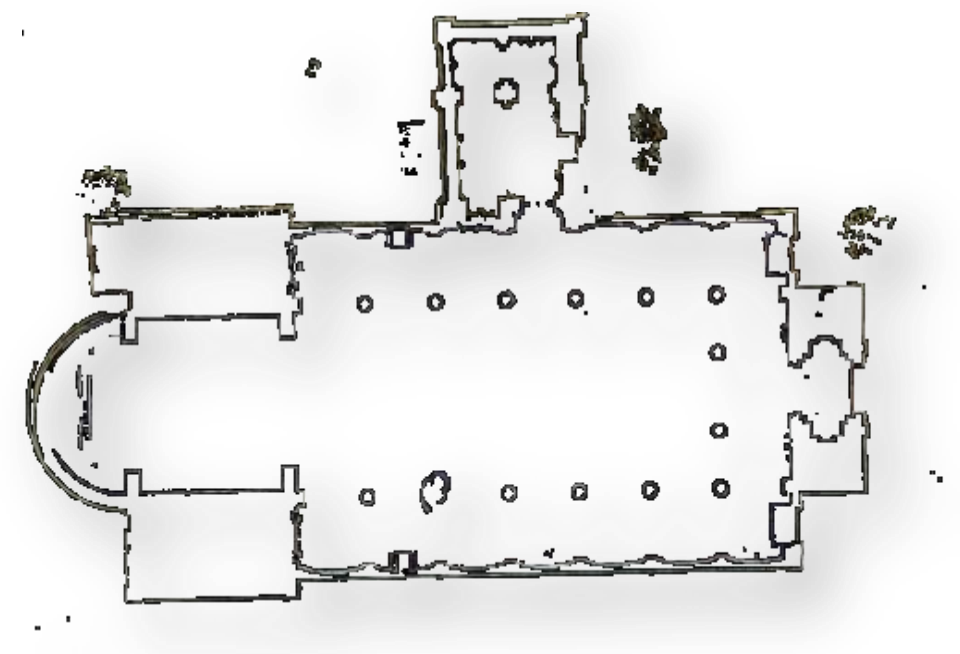

Fig. 5: Footprint of the church and bell tower as part of the ancient chapel

Many documents, such as sections and other profiles (Fig. 5) can thus be extracted from the model and provide to historians the important benchmarks for their investigations on the positioning of the historic building.

This paper shows an alternative modeling process using CAD tools, digitization and reconstruction in single one station point clouds and proposes a method for geometric consolidation to obtain a definitive 3D model. 